\title{
Research on the design of multi-layer steel structure module and steel frame composite building structure
}

\author{
Chi Zhaona * \\ Rizhao Polytechnic, 276826, Rizhao, Shandong, China
}

\begin{abstract}
The composite of multi-layer steel structure module and steel frame, as an important design form in Chinese house construction, is widely used in the architectural design of houses. The multi-layer steel structure module and steel frame composite building structure are different from the traditional. This kind of building mechanism adopts the prefabricated type, which has the advantages of fast construction speed and few limiting factors, and plays a very important guiding role in modern building construction. Based on this, this article will analyze and compare the similarities and differences between steel structure modular buildings and traditional steel frame structures, and explore the design of multi-layer steel structure modules and steel frame composite building structures, hoping to be helpful to relevant people.
\end{abstract}

\section{Introduction}

In the design of multi-layer steel structure module and steel frame composite building structure, it is necessary to use computer technology to design. The construction period of the building project is long and the structure design is complicated. Therefore, in the design of multilayer steel structure modules and steel frames conforming to the structure of the building, the safety of construction personnel must first be focused on. On this basis, it should give full play to professional technology to design a reasonable and effective building plan, optimize the building structure design system, nodes, construction connections and balance the hand relationship of the nodes, etc., rationally choose steel, and reduce the amount of steel structure materials [1].

\section{Targeted selection of structural systems}

If the multi-layer steel structure is used for modular construction, the most basic step is to make the structural steel module reasonably in the corresponding factory, and then transport the formed module to the construction site by targeted means of transportation. For the problems that may occur in the transportation, it is necessary to effectively measure the specification of the module.

For example, technical personnel should start from the relevant national norms and develop a modular structural system plan in accordance with the national norms. For example, in the Code for Building Structure Load, it is stipulated that 'in the module unit, in order to ensure the seismic capacity of the building, the column section should be large, the beam section intersecting the column should be small, and the column section should be large, and a system of strong column and weak beam should be selected'. Therefore, the design document of the designer's modular structural system should include the selection provision of this module system [2]. On this basis, the module is modified so that it can meet the requirements of transportation and construction. At the same time, the modules need to be strictly checked after the production is completed. The technicians can take random sampling from the same batch of modules to test the modules. If the specifications of the modules do not meet the specific specifications of project management, then this batch of modules needs to be returned to the site to prevent production accidents at the source. Specifically, due to the particularity of the construction use of threestory entertainment and leisure building, targeted production of modules is needed. Finally, the height of the module can be controlled below four meters, and for the width of the module, the requirements are not detailed. Designing the width of the module within the range of three to four meters can ensure the functionality of the building play well. This building can effectively adapt to the basic requirements of multi-layer steel structure modules and steel frame composite building structures. The steel frame is used for construction on the overall structure of the three-story building to ensure the strength of the structure to the greatest extent and prevent accidents. In terms of specific details, steel structure modules can be used for effective adaptation, which can effectively improve the overall construction efficiency of the project, and on the basis of ensuring the quality of the project, it can also adjust the construction materials. Therefore, the 
structure is suitable for better application in large-scale leisure buildings.

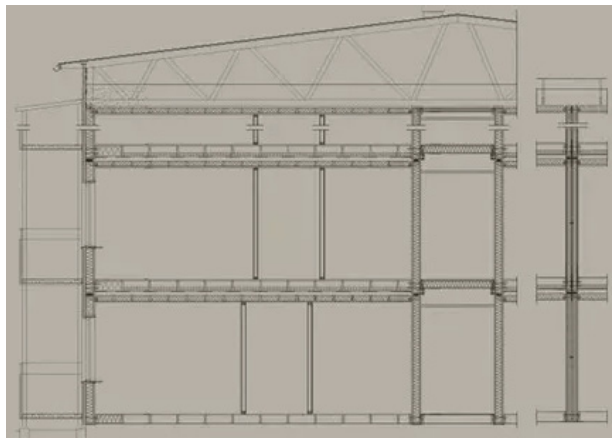

Fig. 1. Modular design of a university library

\section{Node design between modules}

Although welding has a certain technical basis, it should be banned for the long-term development of buildings, so that more safe and reliable structural connection can effectively replace welding links and promote the development of building reliability.

For example, technicians can take a more reliable way to connect the nodes of the steel structure. It can analyze from the basic structure of a multi-layer steel structure building, and use corner fittings to connect the designed nodes. The use of corner pieces can well enhance the stability of the equipment connection, and at the same time it means that the use of welding can be minimized during construction. In the selection of corner fittings, high-strength corner fittings should be the main choice. Different corner fitting strengths should be selected according to different situations, so that the corner fittings can effectively ensure that the steel structure and its corresponding plates are well connected at the same time. It is also necessary to conduct a more scientific and reasonable calculation of the shear resistance between the beam webs so as to better ensure the quality of the project. At the same time, when hoisting, it is necessary to start from the original hoisting sequence, orderly connect each corner piece, and at the same time to reasonably fix the connected corner pieces. When choosing specific steel, it is also necessary to use lighter steel as much as possible, which is also an important step in the architectural design.

At the same time, if corner fittings are not applicable, it should proceed from the specific situation and effectively use welding methods to connect the nodes. First of all, the width of the weld should be measured appropriately before welding, so that the weld can be well adapted to the size of the electrode. The material of the weld must be well adapted to the material to be welded to ensure the unity between the two [3]. When selecting the welding rod, it is necessary to select a welding rod with a relatively low strength for welding, so as to reduce the gradual expansion of the weld. In the process of welding, it is necessary to make the center of gravity of the weld and the center of gravity of the related construction closer.
In this way, the welding success rate between the two can be better improved.

\section{Component design of steel frame}

In the design of the multi-layer steel structure module and the steel frame in line with the building, the steel frame column of the building structure needs to be considered first, and the stress conditions must be fully considered to ensure that the building steel frame has strong stability and bearing capacity, thereby improving the building structure performance.

For example, in the floor design of buildings, it is necessary to ensure the stability of the floor structure at first, and vertical loads can be used to distribute. To distribute the pressure of the floor throughout the building structure, I beam or $\mathrm{H}$ beam can be used to increase the maximum pressure. When the pressure resistance is certain, the seismic design of the whole floor is carried out, and the combination of the pressed steel plate and the castin-place concrete is adopted to form the composite steel frame building structure. In the design process of the architectural structural support system, it is necessary to optimize the axial support of each component in the architectural system [4].

In addition, special treatment is needed for the gaps of the doors, windows and other parts of the building to improve the rationality and implementability of the entire building structure design, and improve the stability of the multi-layer steel structure module and the steel frame system. In the design node of the steel structure, it is first necessary to set up a supporting structure to support the frame evenly in the direction to meet the rigidity requirements of the frame structure. Secondly, it is necessary to design the frame structure in accordance with the design standards of the steel structure module and the steel frame to reach the standard steel structure axial compression stability coefficient. In the design of steel frame structures, the seismic capacity of the entire support system is required to reach $30 \%$ of the seismic capacity of the bottom of the structure. The maximum shear strength needs to be 1.8 times that of the steel structure, which meets the basic standard of frame structure calculation. In the actual steel structure design, the columns of the steel frame structure need to be designed at right angles, which is conducive to the layer displacements in different directions and meets the requirements of the steel structure welding section [5].

In the process of welding steel columns, local thickening can be used, and its range should be controlled, so as to improve the feasibility of the steel structure welding. In the structure design of a multi-layer steel structure module and a steel frame composite building, it is necessary to closely connect each node in the structure to improve the quality of the steel structure. For example, in the design of the beam web, because the beam web needs to bear all the shear forces of the beam, it is necessary to connect the beam web and the column together, which makes it easier to calculate the shear force of the beam. In addition, designers also need to optimize the bearing capacity of the steel structure by combining 
the total span of the beam end and the bending moment forces in different directions. It aims to better facilitate the calculation of the plastic modulus of the beam, improve the accuracy and scientificity of the calculation, and improve the performance of the multi-layer steel module and the steel frame composite structure.

\section{Key points of module design}

In the design of multi-layer steel structure module and steel frame composite building structure, it is necessary to optimize the module design, improve the utilization efficiency of building materials, ensure the connection of nodes in each module can meet the basic requirements, and ensure the rationality and scientific nature of module size and structure design.

For example, in the design of frame structure, it is first necessary to follow the design standard of frame steel structure and clarify the design goal. In the actual construction process, standard construction operations are carried out according to the modular design plan, thereby saving the design cost of the building structure. In modular design, first consider the flexibility and turnover of the steel frame, clarify the design points of the modular design, comprehensively study and analyze the steel frame structure system, and reflect the multi-layer steel structure of the connection nodes and installation conditions. The characteristics of modular design improve the usability and aesthetics of building, and reflect the advantages of multi-layer steel structure modules and frames in conformity with structural design [6].

\section{Strengthen the design details of steel structure and frame structure building}

When designing steel structure modules and steel frame structure composite buildings, the overall design should be effectively controlled. On the basis of ensuring that the overall design direction does not cause problems, the details must be better controlled to prevent it from accidents.

First, the technicians can design the supporting facilities of the building first. For the complicated problems of the supporting pipelines, they should carry out targeted design by adopting the method of concealing. This can better prevent the crossing of different pipelines, and at the same time, the pipeline layout can be carried out without delaying the effective construction of the overall structure, which helps to effectively control the overall construction period. It can further realize the scientific promotion of the actual layout of the building. Secondly, during the process of concealing, the wall decoration laid outside the pipeline can also be controlled in a targeted manner, which helps to minimize the substantial impact on other construction. When grooving work, it needs to choose professional tools and use more professional tools for grooving to enhance the efficiency of the cutting. Thirdly, the selection of materials also needs to meet the corresponding requirements. When the corresponding structure is punched, it can be quickly restored. At the same time, the use of better quality materials can also make the overall waterproof performance a better promotion.

Specifically, in view of the problem of building water seepage, in addition to using good materials, more effective measures need to be taken to make the wall leakage and water seepage conditions effective. For example, while selecting waterproof materials, waterproof coatings can be laid on the exterior of the building to better promote the waterproof performance. The analysis of the characteristics of the composite building with multi-layer more structural modules and steel frame shows that its thermal insulation is not very good. This is very unsuitable for effective heat preservation in the cold winter. When selecting materials, it must choose heat preservation and heat insulation materials for construction.

\section{Conclusions}

In summary, the multi-layer steel structure module and steel frame composite building structure is important in the design of modern building structures. This paper studies and analyzes the design nodes and components in the multi-layer steel structure module and steel frame conforming structure. It can be found that in the design process of the building structure, it is necessary to reasonably select the steel structure module and the steel frame, optimize the joints, and improve the multi-layer steel structure and steel according to the specific construction requirements, considering the size of the module and the characteristics of the composite structure. The frame conforms to the design level of the structure and improves the stability of the steel structure building.

\section{References}

1. Feng Miao, Yang Kewei, Yu Yan. The construction of the teaching experiment of photosensitive nanocomposite intelligent discoloration effect[J]. Experimental Technology and Management, 036.(2019)

2. Ruan Xiaoguang, Cai Dongbao, Ye Xiangdong. Preparation and application of PDMS/SiO2 nanocomposite materials[J]. Applied Chemical Industry, 2018.

3. Tang Tao, Chu Chong, Mao Wenjing. Application research progress of polypyrrole/Cellulose Nano nanocomposites $[\mathrm{J}]$. Chemical Industry Management, 508. (2019).

4. Wang Zhiyou. Research on the design of multi-layer steel structure module and steel frame composite building structure[J]. Engineering Construction and Design. (2019).

5. Zhang Baizhen. Design and research of multi-layer steel structure module and steel frame composite building structure[J]. Science \& Technology Innovation and Application. (2018) 
6. Zhu Liguang. Discussion on the design of multi-layer frame structure based on industrial and civil buildings[J]. Green Building Materials. (2018). 